\title{
CALCULATIONS OF THE TRANSVERSE SPATIAL DISTRIBUTION OF NE102 SCINTILLATION LIGHT MADE BY RECOIL PROTONS FROM COLLIMATED 14-MeV NEUTRONS
}

D. R. Goosman

January 19, 1979

Work performed under the auspices of the U.S. Department of Energy by the UCLLL under contract number W-7405-ENG-48.

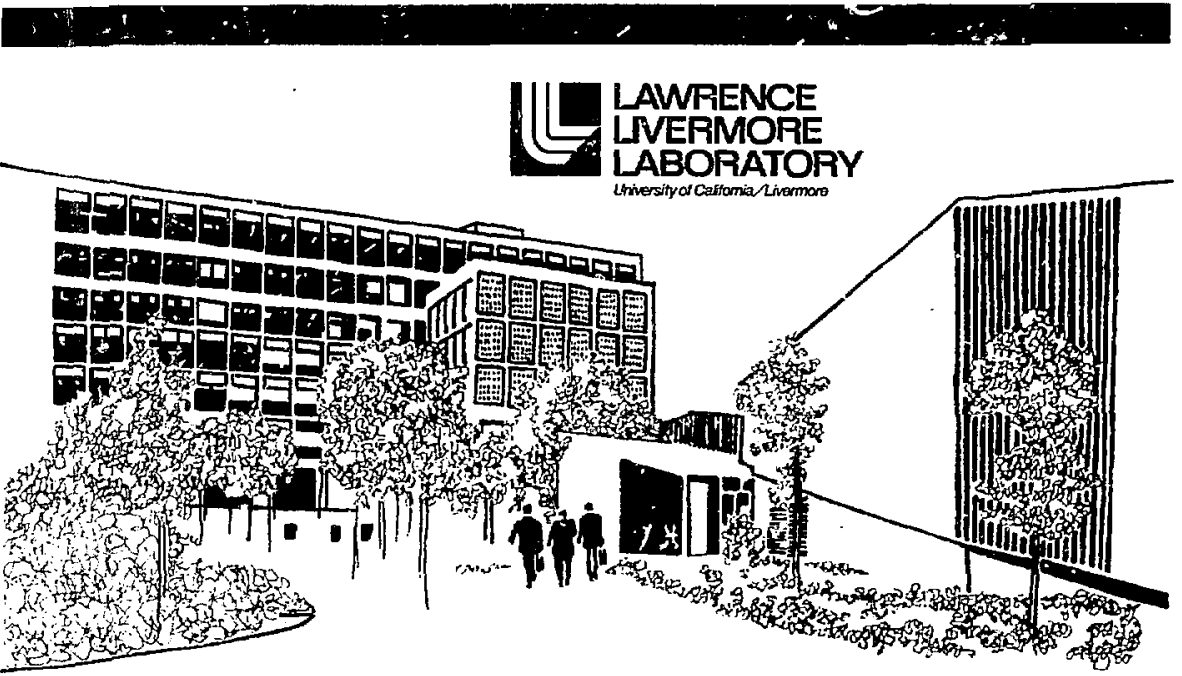

$-19$ 


\section{近}

\section{LAWRENCE LIVERMORE LABORATORY}

University of Caltrmia Livermore, Calfornia 94550

UCRL-52651

\section{CALCULATIONS OF THE TRANSVERSE SPATIAL DISTRIBUTION OF NE102 SCINTILLATION LIGHT MADE BY RECOIL PROTONS FROM COLLIMATED 14-MeV NEUTRONS}

D. R. Goosman

MS. date: January 19,1979

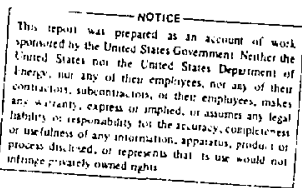




\section{CONTENTS}

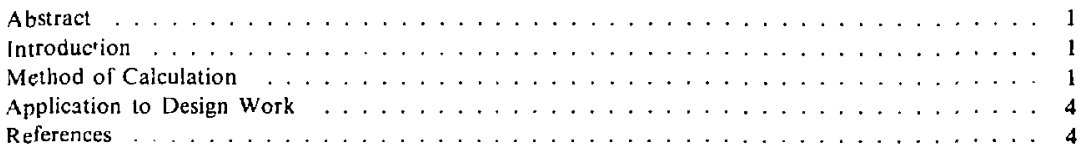




\section{CALCULATIONS OF THE TRANSVERSE SPATIAL DISTRIBUTION OF NE102 SCINTILLATION LIGHT MADE BY RECOIL PROTONS FROM COLLIMATED 14-MeV NEUTRONS}

\section{ABSTRACT}

A calculation has been made of the spatial distribution of NE1( 2 scintillation light produced by recoil protons from collimated 14-MeV neutron illumination. Results were then averaged over offset circles of various radii, thus determining the fraction of the totai scintillation light created inside cylindrical scintillators for uniform neutron illumination over the front face of the scintillator. The results are useful in sensitivity considerations for neutron-imaging camera design.

\section{INTRODUCTION}

Spatial information emanating from thermonuclear plasmas in the form of neutrons can be imaged onto detector arrays consisting of many cylindrical scintillator fibers. Scintillation light from this array can then be coupled to an image intensifier and the result recorded on film. In designing a detection system, the considerations of teriporal and spatial resolution, statistical efficiency, and sensitivity determine the actual dimensions of the scin- tillators. For sensitivity corsiderations, it is useful to know what fraction of the scintillation light made by the neutrons is created inside cylinders of various diameters, assuming uniform spatial neutron itlumination over the front face of the cylinder. This has been calculated, using the experimental results of scintillator light vs proton energy of Craun and Smith. ${ }^{1}$

\section{METHOD OF CALCULATION}

One complication in calculating the fractional scintillation created inside a cylinder of arbitrary diameter is the fact that the light emitted depends upon $\mathrm{dE} / \mathrm{dx}$ in a nonlinear way. A recoil proton slowing down from 14 to $13 \mathrm{MeV}$ produces more light than a proton slowing down from 7 to $6 \mathrm{MeV}$. Therefore, using a Monte Carlo neutron-proton

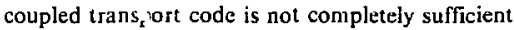
for the calculation if anly the energy deposition is edited, since energy deposition does not uniquely determine light generation. In this paper, a different appruach has been used incorporating the expeimental results of Ref. 1 for the total light produced by the complete stopping of protons of various initial energies.
In order to proceed, several assumptions must be made. The first is that the major source of the scintillation light is due to neutron-proton scattering; the small contributions to the light due to ${ }^{12} \mathrm{C}$ recoils and alphas from ${ }^{12} \mathrm{C}\left(\mathrm{n}\right.$, alpha) and ${ }^{12} \mathrm{C}(\mathrm{n}$, $\mathrm{n} 3 \alpha$ ) are neglected. They are small because of their very high $\mathrm{dE} / \mathrm{dx}$ and low cross section. Multiple interaciions are negligible because the diameters of the cylinders considered are very small compared to a neutron mean-free path.

The second assumption is that the recoil protons are isotropic in the center-.jf-mass system. This is experimentally correct to about $4 \%$ for $14-\mathrm{MeV}$ neutrons. ${ }^{2}$ This means that in the laboratory system the number of recoil protons per unit polar angle is given by 
$\mathrm{dN} / \mathrm{d} \theta=\sin (2 \theta)$

In addition, it is assumed that the recoil protons travel in straight lines in the scintillator material, whereas in reality they straggle transversely somewhat. The cylinders are assumed to be long compared to a $14-\mathrm{MeV}$ proton range $(0.24 \mathrm{~cm})$.

Before the desired fraction of created light can be calculated, it is necessary to calculate the transverse spatial distribution of scintillation light for the cuse of a large number of $14-\mathrm{MeV}$ neutrons entering the scintıliator in the same direction and at the same point. Figure $t$ illustrates this first problem. The neutron enters the scintillator parallel to the horizontal axis and undergoes an n.p elasticscattering event at the origin of coordinates. Recoil proton trajectories in NEl02 with a density of 1.032 $\mathrm{g} / \mathrm{cm}^{3}$ are shown in $5^{\circ}$ steps fis:s 0 to $90^{\circ}$. The proton energies are given by $\mathrm{E}(\theta)=14 \cdot \cos ^{2}(\theta)$. and the ranges were calculated by integrating $\mathrm{dE} / \mathrm{dx}$ data of Ref. I. It is seen from Fig. I that the maximum transverse range is about $0.071 \mathrm{~cm}$. The number of recoil protons per unit $\theta$ is given by the nu:nber indicated at the end of the proton trajectory.

The region of Fig. $I$ is then divided inio 17 cylindrical zones concentric with the horizontal axis. The first 4 zones are $0.0025-\mathrm{cm}$ thick and the latter 13 are $0.005-\mathrm{cm}$ thick. For each proton trajectory, the amount of scintilation light produced in each cylindrical zone is then calsulater. This is accomplished by first combining the proton energy vs totul light produced and proton energy vs range curves from Ref. 1 into a proton range vs light curve. which is shewn in Fig. 2. This curve is more linear than either of the other two curves used to construct it. With this range-light curve it is a simple matter for each proton trajectory to calculate the points of intersection of the trajectory and each cylindrical zone, the residual range of each intersection point, 'nd therefore the light produced in each zone. The results are then weighted by $\mathrm{dN} / \mathrm{d} \theta$ and the resulis for a given transverse zone summed over the contributions from the 19 trajectories. The total light is then divided by the annular area of the zone to produce the light-per-unit-area histogram shown in Fig. 3 and Table 1.

This curve approaches infinity near zero radius, because it was assumed that the protons all arise from the same point and all travel in perfectly straight lines. In reality there is transverse straggling. The fraction of this light distribution contained in a cylinder of indicated radius is also shown in Fig. 3. This curve applies only for on-axis neutron illumination.

For most situations, one deals with uniform neutron illumination over the entire front face if the cylinder. Thus, the distribution of Fig. 3 must be averaged over circles of various diameters. This has been done numerically with Monte Carto tech. niques and the result is shown in Fig. 4. For example, a long scintillator $0.2 \mathrm{~cm}$ in diameter illuminated uniformly with $14-\mathrm{MeV}$ collimated

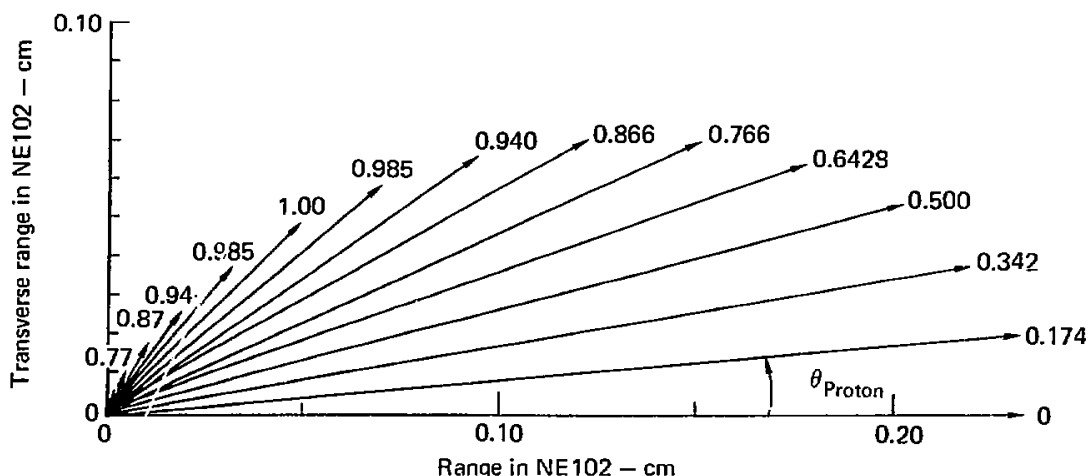

FIG. 1. Geometry of the problem, showing the proton trajectories in $5^{\circ}$ steps. The NE102 density is 1.032 $\mathrm{g} / \mathrm{cm}^{3}$. 


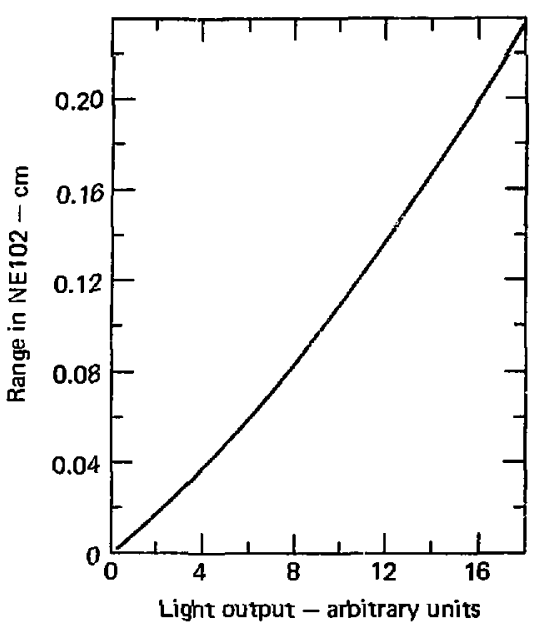

FIG. 2. Deduced proton range vs total scintillation itght.

neutrons parallel to its axis has, on the average, $81 \%$ of the total scintillation light produced within its boundaries.

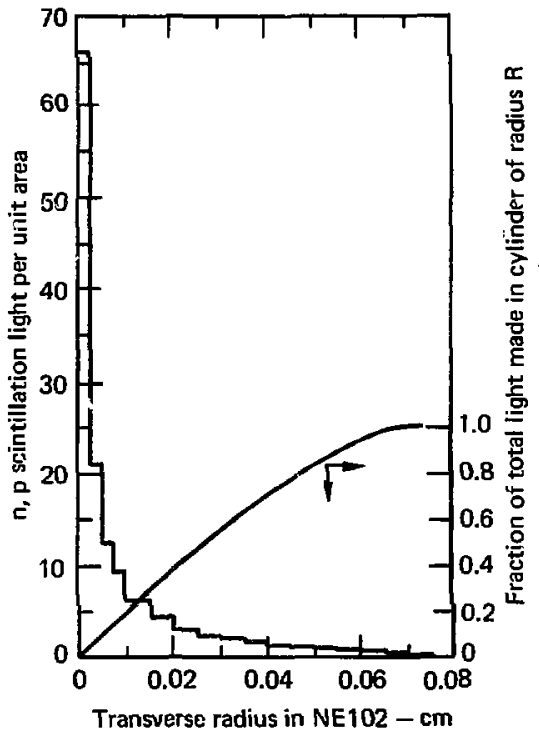

FIG. 3. The distribution of scintillation light in NE 102 transverse to the $14 \mathrm{MeV}$ neutron direction. The NE102 density is $1.032 \mathrm{~g} / \mathrm{cm}^{3}$.

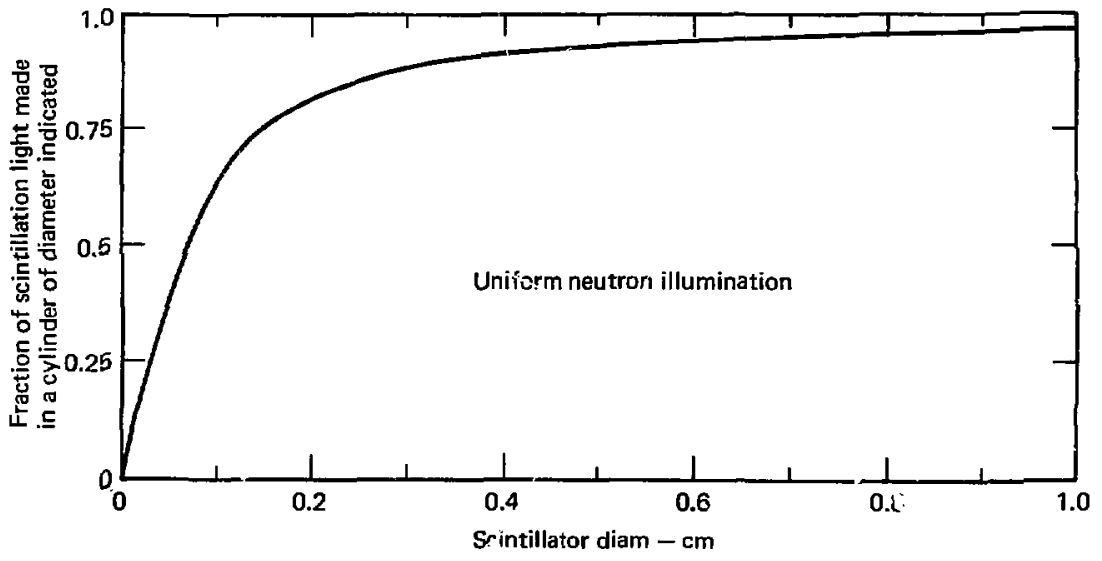

FIG. 4. The fraction of scintillation light made in cylinders of the diameter iudicated for uniform neuiron illumination. 
TABLE !. Tabular representation of Fig. 3.

\begin{tabular}{lcc}
\hline $\begin{array}{c}\text { Inner radius, } \\
\text { cn }\end{array}$ & $\begin{array}{c}\text { Outer radius, } \\
\text { cm }\end{array}$ & $\begin{array}{c}\text { Scintillation light } \\
\text { per unit area } \\
\text { (arbitrary units) }\end{array}$ \\
\hline 0 & 0.0025 & 66.2 \\
0.0025 & 0.0950 & 21.1 \\
0.0050 & 0.0075 & 12.5 \\
0.0075 & 0.010 & 9.47 \\
0.010 & 0.015 & 6.23 \\
0.015 & 0.020 & 4.44 \\
0.020 & 0.025 & 3.05 \\
0.025 & 0.030 & 2.37 \\
0.030 & 0.035 & 2.12 \\
0.035 & 0.040 & 1.73 \\
0.040 & 0.045 & 1.35 \\
0.045 & 0.050 & 1.15 \\
0.050 & 0.055 & 1.01 \\
0.055 & 0.060 & 0.767 \\
0.060 & 0.065 & 0.655 \\
0.065 & 0.070 & 0.338 \\
0.070 & 0.075 & 0.012 \\
\hline
\end{tabular}

\section{APPLICATION TO DESIIGN WORK}

Ankenbrandt and Lent ${ }^{3}$ have shown that for a perfect optical-quality cylindrical scintillator with an index of refraction in greater than $\sqrt{2}$, the fraction of the scintillation light produced uniformly within the cylinder that escapes each end (integrated over time) is simply

$$
L_{\text {end }}=1 / 2\left(1-\frac{\sqrt{n^{2}-1}}{n}\right) .
$$

This result is independent of the length and diameter of the cylinder. Thus, for perfect optical- quality scintillarion fibers, one can use this value of $\mathbf{L}_{\text {end }}$ along with the result of Fig. 4 and the absolute scintillation efficiency of a particular scintillator to estimate the light-per-unit area leaving one end of the scintillator for an average single-neutron interaction. In this way, it is possible to estimate if single interactions can be recorded by the film or other recording mechanism. For film recording, one might conclude that the smaller the fiber diameter, the better, but in practice the optical quality of the fibers and the limiting resolution of other elements of the system must be considered.

\section{REFERENCES}

1. R. L. Craun and D. L. Smith, Nucl. Instrum. Methods 80, 239 (1970).

2. W. N. Hess, Rev. Mod. Phys. 30, 368 (1958).

3. C. M. Ankenbrandt and E. Mi. Lent, Rev. Sci. Instrum. 34, 647 (1963). 